\title{
GFAP Delta as Divergent Marker of Human Glial Progenitors
}

\author{
Cristina Zalfa ${ }^{1}$, Chiara Grasselli ${ }^{1}$, Guido Santilli' ${ }^{1}$, Daniela Ferrari ${ }^{1}$, Giuseppe Lamorte ${ }^{2}$, Angelo Luigi Vescovi ${ }^{1,2,3}$ and Lidia De Filippis ${ }^{3 *}$ \\ ${ }^{1}$ Department of Biotechnologies and Biosciences, Universita' Milano Bicocca, Piazza della Scienza, Milan, Italy \\ ${ }^{2}$ Istituto C.S.S. Mendel, Viale Regina Margherita, Roma, Italy \\ ${ }^{3}$ Casa Sollievo della Sofferenza IRCCS, Viale dei Cappuccini, San Giovanni Rotondo (FG), Italy
}

\begin{abstract}
Background: Human Neural Stem Cells (hNSCs) are responsible for brain development at prenatal and postnatal stages and for tissue homeostasis and repair after injury on adulthood. The identification of hNSC specific markers is still a debated argument. GFAP-ס, the delta isoform of Glial Fibrillary Acidic Protein (GFAP), is particularly expressed in the Subventricular Zone (SVZ) of the brain and its expression has been related to long-term quiescent NSC. On the other hand, another cytoskeletal protein, gelsolin, is expressed in Neural Progenitor Cells (NPCs) migrating from the SVZ.
\end{abstract}

Methods: In this study, we intended to investigate by immunocytochemistry the co-expression of GFAP- $\delta$ and gelsolin in different sources of hNSCs, and hNPCs, with particular emphasis on Good Manifacture Procedures (GMPs) grade hNSC currently used in two clinical trials on Amyotrophic Lateral Sclerosis (ALS) and Multiple Sclerosis (MS) patients.

Results: We found that the two proteins are co-expressed by undifferentiated GFAP+ cells but tend to localize in different sub-cellular compartments and to segregate in divergent GFAP+ progenies along with differentiation. Interestingly, we proved that, after transplantation into the brain of rodent models of focal demyelination or transient global ischemia, hNSC integrating in the SVZ still retain the co-expression of GFAP- $\delta$ and gelsolin, indicating that hNSC intrinsically co-express the two proteins at the stem stage.

Conclusion: These findings suggest that GFAP- $\delta$ and gelsolin may represent two candidate markers to distinguish NSC from NSC-deriving divergent astroglial phenotypes.

Keywords: Neural stem cells; Glia; GFAP; Gelsolin; Glial progenitors; Neurodegenerative diseases

Abbreviations: hNSCs: Human Neural Stem Cells; SVZ: Subventricular Zone; SGZ: Subgranular Zone; NPCs: Neural Progenitor Cells; ALS: Amyotrophic Lateral Sclerosis; MS: Multiple Sclerosis; GMP: Good Manifacture Procedure; AD: Alzheimer's Disease; IF: Intermediate Filament; GFAP: Glial Fibrillary Acidic Protein; GalC: Galactocerebroside C; PCR: Polymerase Chain Reaction; PFA: Paraformaldehyde; SEM: Standard Error of Mean

\section{Introduction}

\section{Background}

hNSCs play a key role in neurogenesis, both under physiological and pathological conditions, since they are responsible of tissue homeostasis and repair to brain damage after injury. Though mostly quiescent, they can proliferate under certain circumstances and differentiate into the three main neural cell lineages: neurons, oligodendrocytes and astrocytes. A wide array of studies in progress is still aimed at identifying NSC specific markers, with GFAP and nestin being commonly recognized as non-specific markers of NSC. Indeed, GFAP is also a marker of astrocytes, while nestin of Neuronal Progenitor Cells (NPCs). In neurogenesis, GFAP+ cells include both the stem cell source generating NPCs and astroglial cells providing trophic and instructing support to migrating NPCs and neuronal differentiation. GFAP is a protein of Intermediate Filaments (IFs) which represent a master player in the cytoskeletal organization, cell migration and adhesion, with multiple splicing variants that have been identified and variably related to different areas of expression and functions. In particular, two peculiar cytoskeletal proteins have been associated to NSCs-derived glial cells and related neurodegenerative diseases like Alzheimer's Disease (AD): gelsolin and GFAP- $\delta$.
Human GFAP- $\delta$ is a GFAP protein isoform that GFAP- $\delta$ is encoded by an alternative splice variant of the GFAP gene. Previous studies have shown that GFAP- $\delta$ is specifically expressed by a subpopulation of astrocytes located in the subpial zone of the cerebral cortex, the Subgranular Zone (SGZ) of the hippocampus and by a ribbon of astrocytes following the ependymal layer of the cerebral ventricles. Therefore, GFAP- $\delta$ specifically marks the astrocytes in the SVZ containing the NSCs [1]. Interestingly, its expression has been found upregulated in SVZ of patients of $\mathrm{AD}$, most likely as a consequence of constitutive splicing than of astrogliosis. Indeed, given the ability of GFAP- $\delta$ to change the assembly properties of GFAP filaments, its role has been related to the modulation of the volume and/or cellular location of IFs in astrocytes. Van Den Berge, et al. [2] have also shown that GFAP- $\delta$ is specifically expressed in long-term quiescent cells in the human SVZ.

Gelsolin, a cytoskeletal protein, is a founding member of a family of actin binding proteins involved in controlling the organization of the actin cytoskeleton in cells [3]. Its expression seems to be involved in the regulation of membrane ruffling and chemotaxis [4,5], but it may also act as an inhibitor of $\mathrm{A} \beta$ fibrillization in $\mathrm{AD}$ patients

*Corresponding author: Lidia De Filippis, Casa Sollievo della Sofferenza IRCCS Viale dei Cappuccini, San Giovanni Rotondo (FG), Italy, Tel: +39(0882)410346 E-mail: lidia.defilippis@unimib.it; I.defilippis@operapadrepio.it

Received August 03, 2018; Accepted September 11, 2018; Published September 17, 2018

Citation: Zalfa C, Grasselli C, Santilli G, Ferrari D, Lamorte G, et al. (2018) GFAP Delta as Divergent Marker of Human Glial Progenitors. Stem Cell Res Ther 8: 437 doi: 10.4172/2157-7633.1000437

Copyright: (c) 2018 Zalfa C, et al. This is an open-access article distributed under the terms of the Creative Commons Attribution License, which permits unrestricted use, distribution, and reproduction in any medium, provided the original author and source are credited. 
and as an antioxidant and anti-apoptotic protein [6]. In particular, cytoplasmic gelsolin (c-gelsolin) has been shown upregulated in cells under oxidative stress through a mechanism involving PKC activity as determinant for its upregulation. Moreover, Kronenberg, et al. [7] have demonstrated that gelsolin deficiency does not affect proliferation or neuronal differentiation of adult NPCs but causes a delay of their migration ability in vitro, consistently with slowing the emigration of newly generated cells from the SVZ to the olfactory bulbs in vivo.

Considering that the morphological changes mediated by cytoskeletal dynamics are associated to the neural differentiation of NSC and their migration through the physiological neurogenic pathways and, at last instance, to aging and cell death, we analysed the co-expression of gelsolin and GFAP- $\delta$ in human neural cell lines both in vitro and in vivo. In particular, we investigated the expression and localization of GFAP- $\delta$ and gelsolin in different sources of NPCs and found that the two proteins tend to segregate in separate subcellular compartments and, with differentiation, in different astroglial cells; this dichotomy was maintained in vivo after transplantation of hNSC in the SVZ of healthy such as in lysolecitine or ischemia lesioned rats. In this study, we show how GFAP- $\delta$ and gelsolin are co-expressed in undifferentiated cells but never co-localize in the same structure and we hypothesize they could have specific and complementary roles in stem cell differentiation and migration.

\section{Methods}

\section{Primary cultures of neural cells from human cortex}

Healthy frontal cortex tissue was obtained from fully anonymous leftover material exempt from the San Gerardo Institutional Review Board (IRB) approval as per Hospital regulations (ASG-DA-050 Donazione di materiale biologico a scopo di ricerca e/o sperimentazione, May 2012).

Tissues were dissected and digested in a papain solution (Worthington Bio- chemical, Lakewood, NJ, USA). Primary cells (acutely isolated cells) were plated in DMEM basal medium containing $10 \%$ fetal calf serum and cultured for 10 div before immunocytochemical analysis.

Immortalization of hNSC and $\mathrm{MeV}$ was performed as described by De Filippis, et al. [8].

\section{Differentiation of hNSC and immunocytochemistry}

hNSC were derived from telencephalic-diencephalic or from the mesencephalic region of human foetal brain from abortive tissues and cultured as neurospheres in compliance with GMP guidelines [9].

hNSC ( $n=2$ lines) were cultured by neurosphere assay as described by De Filippis, et al. [8]. hNSCs were cultured in 5\% oxygen and at passage 15 they were splitted to $1 \%$ and $5 \%$ oxygen. In order to allow the adaptation to the new oxygen concentrations, hNSCs were cultured for $4-5$ passages in $1 \%$ and $5 \%$ before starting all the analysis shown in the paper. Therein respective exposures of hNSCs to different oxygen concentrations were continuously maintained in two different humidified $\mathrm{CO}_{2}$ multigas incubators (CELBIO), flushed continuously with a $\mathrm{N}_{2}$ gas to maintain established $\mathrm{O}_{2}$ concentrations (1\%, and $5 \%$ with respect to atmospheric pressure) at a constant temperature of $37^{\circ} \mathrm{C}$.

Astrocytes were derived from primary cortex NPC or hNSC through 2-3 passages on adhesive support in the culture medium containing FGF2 and 2\% FCS, followed by terminal differentiation for 10 days in vitro (div), in 10\% FCS basal medium. The presence of non- astroglial cells in the culture, e.g., neurons and oligodendrocytes, was monitored by immunostaining with specific neuronal ( $\beta$-Tubulin III) and oligodendroglial (Galactocerebroside $\mathrm{C}(\mathrm{GalC})$ ) markers. Sporadic neurons and no oligodendrocytes were detectable in the culture [10].

hNSCs were differentiated as described by Middeldorp, et al. [11] under different treatment conditions. Cells were fixed in $4 \%$ Paraformaldehyde (PFA) at $0,3,10$ or 17 days along differentiation and processed for Western Blot (see below) or immunocitochemistry (11) with primary (Table 1) and secondary antibodies (Table 2). Microphotographs were taken using a Zeiss Axiovert 200 direct epifluorescence microscope or with confocal microscope NIKON ECLIPSE Ti.

\section{Quantitative Polymerase Chain Reaction (Q-PCR)}

For RNA isolation $1 \mathrm{ml}$ of TRIzol reagent (Invitrogen) was added to the human fetal neurosphere pellets. Total RNA isolated according to the manufacturer's protocol was further processed for Q-PCR as described by Dijk, et al. previously [12]. Sequences of the used primers are shown in Table 2. The resulting Ct values were converted to absolute amounts of cDNA present in the sample (E-Ct) [13]. To correct for differences in cDNA amounts between samples, the target PCR was normalized to the geomean values of PCRs on a set of reference genes, i.e., E2 ubiquitin ligase (E2Ubi), RNA polymerase II (RNA pol II), and $18 \mathrm{~S}$ ribosomal RNA (18S) were most stable and were used for normalization.

\section{Western blot analysis}

Immunoblots were performed as described by De Filippis, et al. [8]. Proteins were revealed by chemioluminescence (ECL, Amersham Biosciences $\mathrm{AB}$ ) and detected on an X-ray film (Amersham Biosciences $\mathrm{AB})$. Antibodies listed in Supplementary Table S1. Image processing and densitometric quantification were performed with using Image J.

\section{Animal studies}

hNSCs were injected into the corpus callosum of adult rats at 5 days from lysolecitine-induced lesion $(n=3)$ (Sprague Dawley, Charles River, USA) as described by Rota, et al. [14] and into the hippocampal fissure of adult rats at 5 days from global transient ischemia $(n=3)$ as

\begin{tabular}{|c|c|c|c|}
\hline \multicolumn{4}{|l|}{ Immuno-Fluorescence } \\
\hline Antigen & Supplier & Code & Concentration \\
\hline$\beta$-tubulin III ( $\beta$-tubllI, mouse) & Covance & MMS-435P & $1: 400$ \\
\hline $\begin{array}{l}\text { Glial Fibrillary Acidic Protein } \\
\text { (GFAP, mouse) }\end{array}$ & Chemicon & MAB3402 & $1: 500$ \\
\hline Ki67 (rabbit) & Novus Biologicals & NB600-1252 & $1: 500$ \\
\hline $\begin{array}{l}\text { Galactocerebroside C (GalC, } \\
\text { mouse) }\end{array}$ & Chemicon & MAB342 & $1: 200$ \\
\hline $\begin{array}{l}\text { Human Nuclei (hNuclei, } \\
\text { mouse) }\end{array}$ & Millipore & MAB1281 & $1: 100$ \\
\hline Oligodendrocyte marker $\mathrm{O} 4$ & Chemicon & MAB345 & $1: 200$ \\
\hline Gelsolin & Abcam & Ab74420 & $1: 200$ \\
\hline Vimentin & Millipore & MAB3400 & $1: 400$ \\
\hline A2B5 & Cell Signaling & $\# 2225$ & $1: 100$ \\
\hline
\end{tabular}

Table 1: Primary antibodies.

\begin{tabular}{|l|l|l|l|}
\hline Immuno-Fluorescence & Supplier & Code & Concentration \\
\hline Secondary antibody & Molecular Probes & $\begin{array}{l}\text { A11010 and } \\
\text { A-21123 }\end{array}$ & 1:800 \\
\hline Alexa 546 (against rabbit lgG) & M-11008 and & 1:800 \\
\hline Alexa 488 (against rabbit lgG) & Molecular Probes & $\begin{array}{l}\text { A-21121 } \\
\text { A-211 }\end{array}$ &
\end{tabular}

Table 2: Secondary antibodies. 
described by Nielsen, et al. [15]. All animal care and experimental procedures were conducted according to the current national and international animal ethics guidelines and approved by the Italian Ministry of Health.

Immunohistochemistry was performed on cryopreserved brain sections fixed with $4 \%$ PFA. GFAP- $\delta$ and gelsolin expression in hNSCs was assessed through confocal co-localization with the human-specific nuclear antigen, human Nuclei or Ki67.

\section{Statistical analysis}

For immunocytochemistry, data are reported as percentages of labeled cells over the total nuclei \pm the Standard Error of Mean (SEM). An average of $3 \times 10^{3}$ total cells (identified by DAPI nuclear staining) was counted randomly from 2 coverslips per condition in each experiment. Each value represents the average of three independent experiments. Data were analyzed by Student's t-test and one-way ANOVA (Bonferroni test) and reported as mean \pm SEM and are considered not statistically significant unless indicated in the figures $\left({ }^{*} \mathrm{P} \leq 0.05,{ }^{* *} \mathrm{P} \leq 0.01,{ }^{* *} \mathrm{P} \leq 0.001\right)$.

\section{Results}

\section{Expression of GFAP- $\delta$ in hNSC}

GFAP is one of the main IF proteins in astrocytes and the predominant isoform is GFAP- $\alpha$. Isoform $\delta$ [15], produced by alternative splicing of GFAP gene, includes a new tail domain which prevents homodimerization and formation of homomeric filament formation, whereas the ability to form heterodimers and filaments with GFAP- $\delta$ is retained. Other splicing variants of GFAP have been identified by previous studies in pyramidal neurons of $\mathrm{AD}$ and Down syndrome patients [16] and associated to a possible retro-differentiation of AD hippocampal neurons: $\Delta 164, \Delta$ exon 6 and $\Delta 135$. As previous studies [1] have shown that the expression of GFAP- $\delta$ is selectively expressed in correspondence of brain areas that are currently identified as stem cell niches, like the SVZ and the SGZ of hippocampus, we focused our attention on this variant and checked for its expression during the main steps of differentiation. We performed a quantitative analysis to check for the expression of GFAP- $\delta$ in neurospheres, doublets (undifferentiated at 48 hours from neurosphere dissociation) and differentiated cells derived from IhNSC [8]: the m-RNA of GFAP- $\alpha$ correlated with that of $-\delta$ variant and was shown to be upregulated with differentiation. Consistently with a preliminary RT-PCR (Figure 1a) Western Blot analysis showed that the protein amount of GFAP- $\delta$ increases with differentiation (Figure 1b).

Both GFAP- $\delta$ and gelsolin have been previously shown to be expressed in the SVZ [1,7] and related respectively to the long-term quiescence of adult SVZ NSCs and to the NPCs migration along the neurogenic pathways. To test the intrinsic expression of the two proteins in NSC cultured in vitro, thus irrespectively of non-neural components, we approached a preliminary characterization of GFAP- $\delta$ and gelsolin expression in GMP grade hNSC that are currently exploited in a clinical trial on ALS patients [9]. Immunocytochemistry showed that both the proteins co-localize in GFAP+ cells and are expressed in dividing cells (Figure 1d). Cytofluorimetric analysis confirmed the dual expression of both the proteins in undifferentiated hNSC (Figure 1c) and their co-localization in GFAP+ cells (Figure 1e).

\section{Divergent expression of GFAP- $\delta$ and gelsolin in NPC and hNSC lines}

Considering that gelsolin is widely expressed troughout the brain and a weak expression of GFAP- $\delta$ has been observed in the sub-pial cortex [1], we examined both primary culture and astroglial progenitors deriving from adult human cortex (Figure 2a), showing that both GFAP- $\delta$ and gelsolin are detectable in astroglial GFAP+ cells cells even if never co-localizing together. In parallel, we analyzed both mixed (neurons, astrocytes and oligodendrocytes) and astrocyte-enriched cultures obtained from differentiation of fetal NSC (hNSC) deriving from the telencephalic-diencephalic region of fetal human brain [17]. The expression of both the proteins was maintained in astroglial cells of mixed differentiated such as of astrocyte cultures (Figure 2b). Since GFAP- $\delta$ has been recently found expressed in long-term quiescent cells proximal to the SVZ of adult human brain, we performed a further analysis on differentiated progeny from hNSC immortalized with $\mathrm{v}$-myc gene (IhNSC) endowed of an enhanced self-renewal potential with respect to parental hNSC, while NPC deriving from human foetal ventral mesencephalon and immortalized with v-myc (I-hVMe) (Figure 2a) were analyzed as negative control for GFAP- $\delta$ which is normally not expressed in mesencephalon. Interestingly, GFAP- $\delta$ appeared weakly expressed in the processes of IhNSC with respect to hNSC where it clearly appeared to be enwrapped in perinuclear bundles (Figure 2b) besides distributed along processes. On the contrary, gelsolin appeared organized in parallel filaments and more markedly expressed in cells with low or faint expression of GFAP- $\delta$ such as I-hVMe. These results show that gelsolin and GFAP- $\delta$ are expressed by differentiated progeny of hNSC independently on the differentiation process or on their proliferation potential and do never co-localize, supporting the hypothesis that they could exert different functions within the same differentiating progenitor cell and/or represent two selective markers for different astrocyte subtypes.

\section{Expression of GFAP- $\delta$ and gelsolin in hNSCs}

Considering that SVZ astrocytes are commonly accepted as NSCs [18] and that GFAP- $\delta$ was detected in the ribbon of astrocytes proximal to SVZ in the human adult brain, we checked for the coexpression of GFAP- $\delta$ with total GFAP by immunofluorescence either in parental hNSC and immortalized IhNSC [8] during differentiation in vitro. While immunostaining indicated that the relative percentage of GFAP- $\delta+$ cells is higher at the stem stage $(0$ div) or the transient progenitor stage ( 3 div) with respect to the terminally differentiating stages (10-17 div), the amount of GFAP- $\delta$ expression per cell was clearly upregulated from 3 to 10 days of differentiation (Figure 3 ), thus confirming the results from Western Blot (Figure 1b). In particular, GFAP- $\delta$ appeared expressed in the perinuclear region at early stage, while progressively organizing as a filament boundle and through the processes along with differentiation (Figure 3 ) and the majority of GFAP+ cells was also GFAP- $\delta+$. When we checked for co-localization with gelsolin, both GFAP- $\delta$ and gelsolin were co-expressed by astroglial cells but they were progressively segregating in different sub-cellular areas and in different cells over differentiation.

Despite the progressive decrease of Ki67+ proliferating cells over differentiation (Figure 4a), the majority of Ki67+ cells were also GFAP- $\delta+$ (Figure $4 \mathrm{a})$, consistently with previous studies indicating GFAP- $\delta$ expressed in proliferating stem-like cells and glial progenitors during differentiation.

From these results, we could hypothesize that GFAP- $\delta$ could represent a marker for a specific stage in the differentiation of hNSCderived glial progenitors. Accordingly, while no colocalization was detected with the early neuronal marker $\beta$-tubulin III (Figure $4 \mathrm{~b}$ ), GFAP- $\delta$ was detected both in A2B5+ and vimentin + cells such as in $\mathrm{O} 4+$ and GalC+ oligodendroglial cells (Figure $4 \mathrm{~b}$ ), suggesting GFAP- $\delta$ 
Citation: Zalfa C, Grasselli C, Santilli G, Ferrari D, Lamorte G, et al. (2018) GFAP Delta as Divergent Marker of Human Glial Progenitors. Stem Cell Res Ther 8: 437. doi: 10.4172/2157-7633.1000437

a

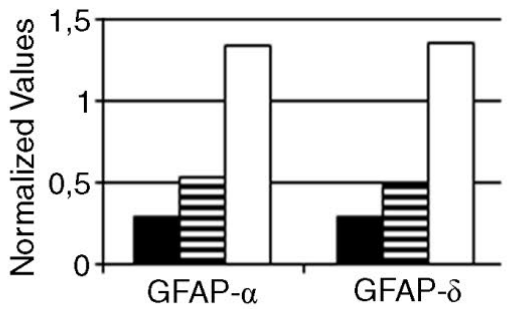

b

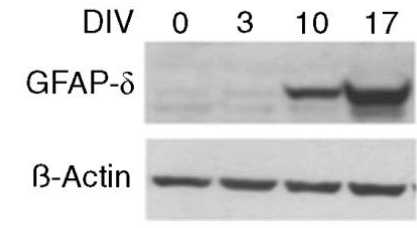

- Stem Neurospheres EStem Doublets םDifferentiated Cells

C
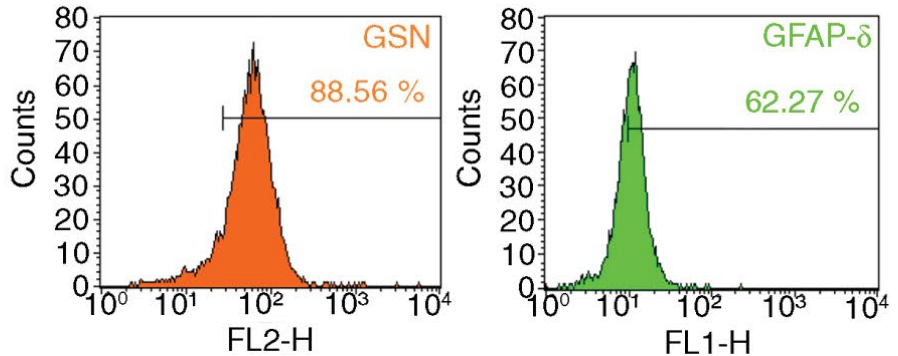

d

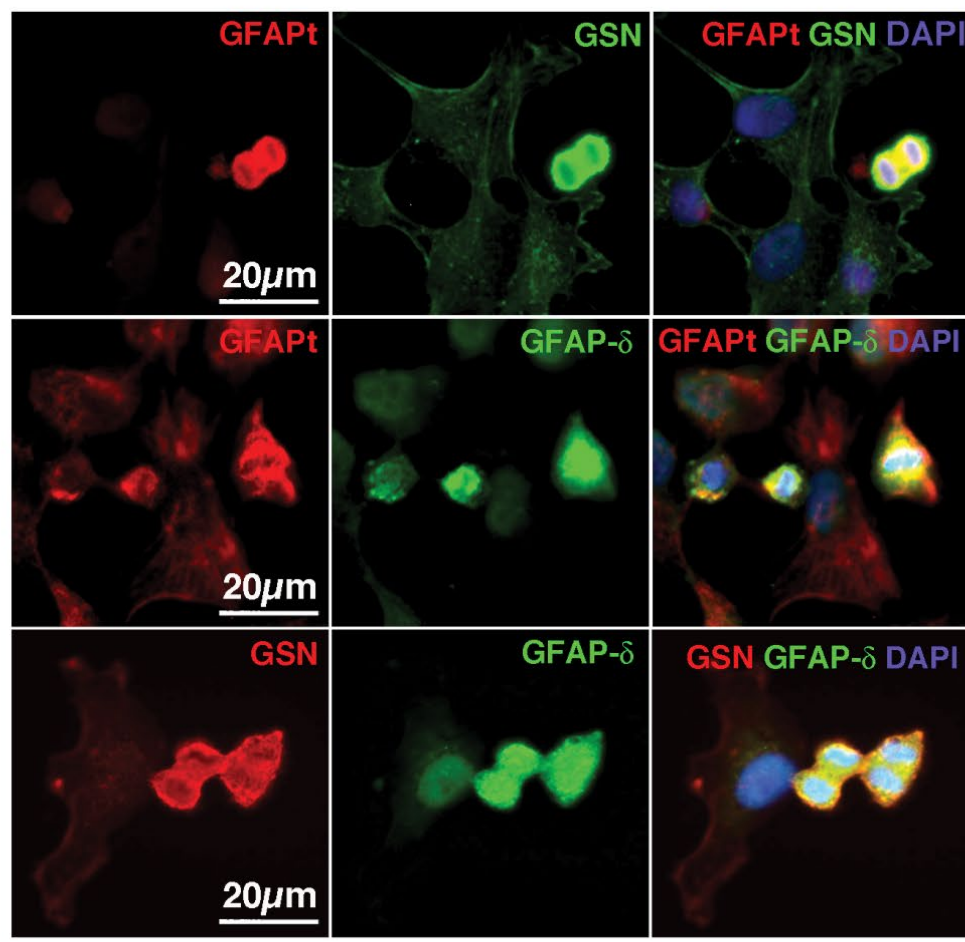

e
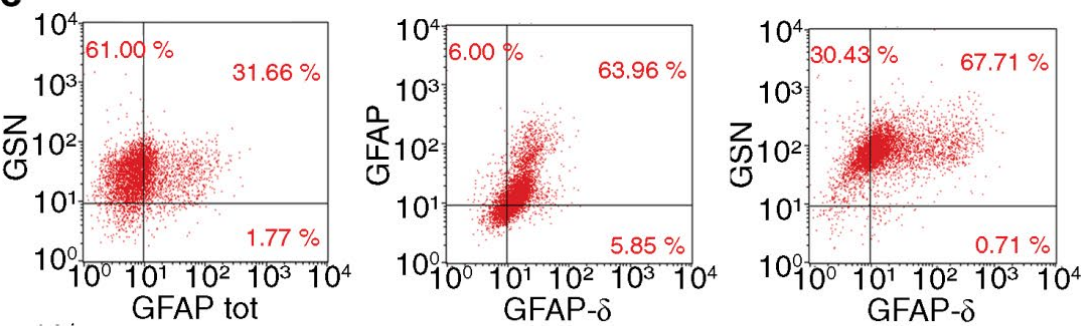

Figure 1: Expression of GFAP- $\delta$ in hNSC. a) qRT-PCR of GFAP- $\delta$ and GFAP- $\alpha$ in hNSC neurospheres, doublets (dissociated neurospheres after 48 hours from dissociation) and differentiated for $10 \mathrm{div}$; b) Western Blot analysis of GFAP- $\delta$ in differentiating hNSC at $0,3,10$ and 17 div (days of differentiation); c) citofluorimetric analysis of GFAP- $\delta$ and gelsolin expression in neurospheres; d-e) immunofluorescence (d) and citofluorimetric (e) analysis showing co-localization of GFAP- $\delta$ and gelsolin, GFAP- $\delta$ and GFAP total, gelsolin and GFAP total in undifferentiated hNSC. Scale bar: $20 \mu \mathrm{m}$. 
Citation: Zalfa C, Grasselli C, Santilli G, Ferrari D, Lamorte G, et al. (2018) GFAP Delta as Divergent Marker of Human Glial Progenitors. Stem Cell Res Ther 8: 437. doi: 10.4172/2157-7633.1000437
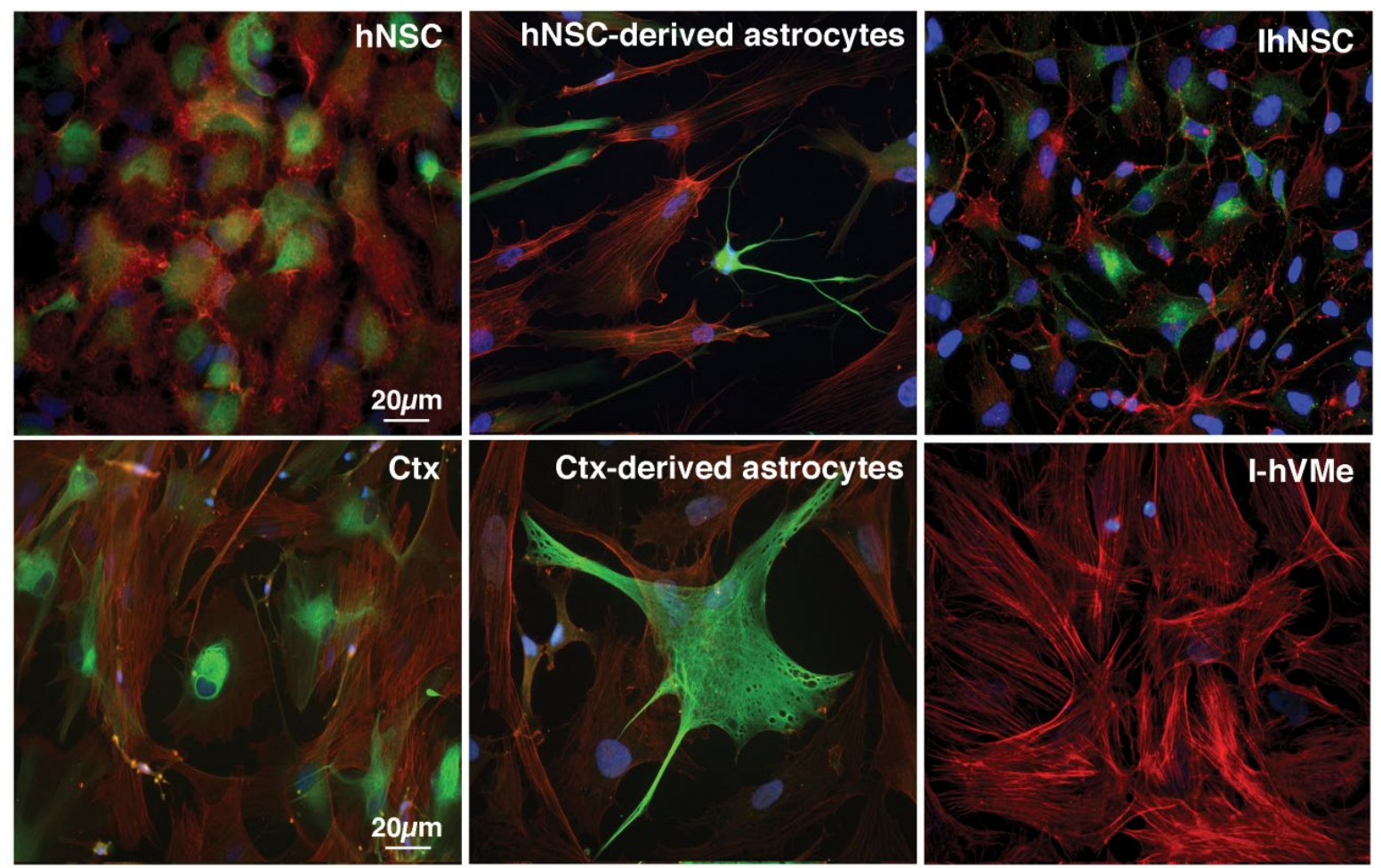

Figure 2: Expression of GFAP- $\delta$ and gelsolin in different cell sources. Immunofluorescence analysis of expression of GFAP- $\delta$ and gelsolin markers in a) Ctx, Ctx-derived astocytes and I-hVMe, and b) hNSC, hNSC-derived astrocytes, IhNSC. Scale bar: $20 \mu \mathrm{m}$.
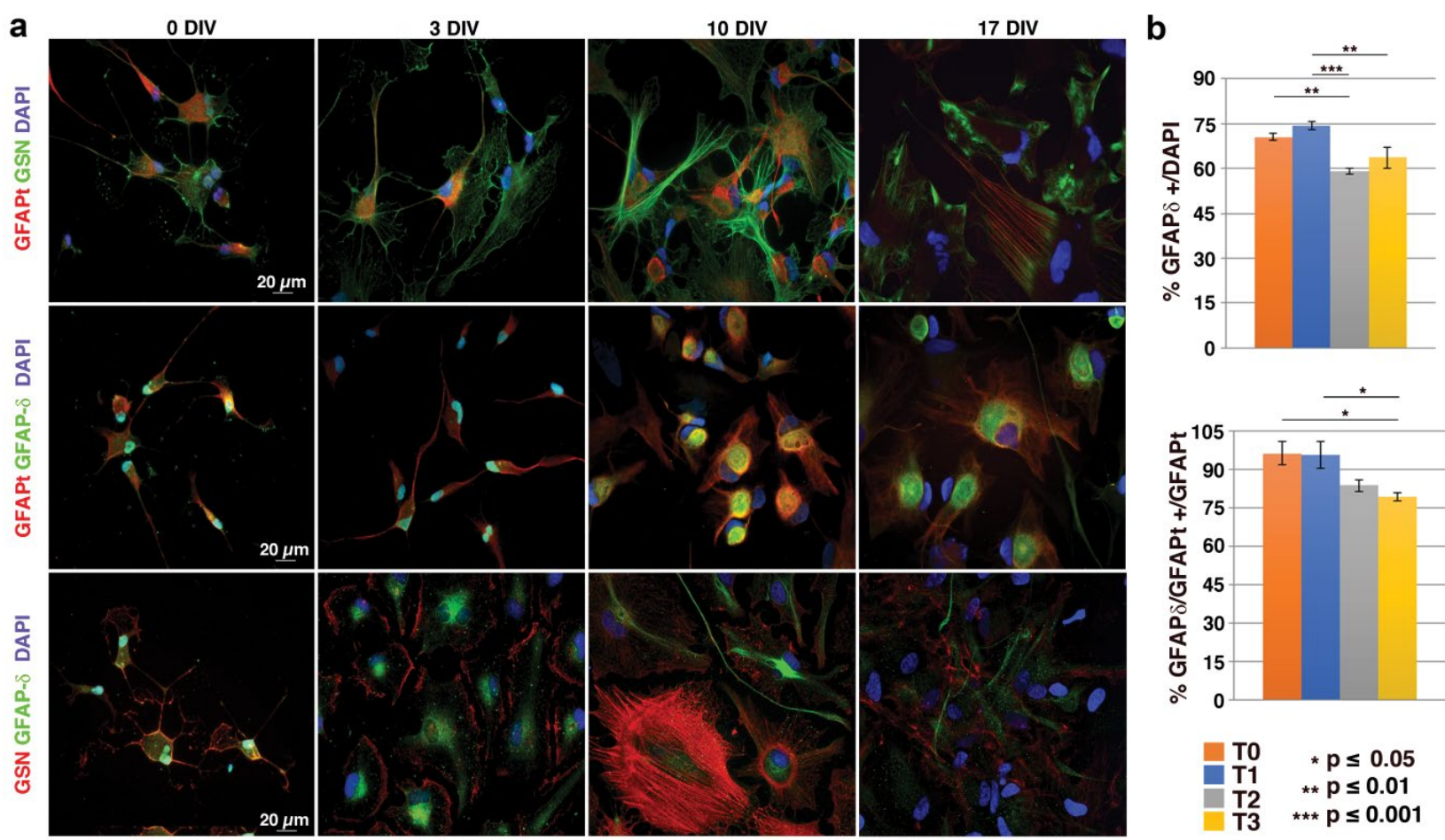

Figure 3: Expression of GFAP- $\delta$ and gelsolin in differentiating hNSC. a) Immunofluorescence analysis of expression of GFAP- $\delta$, total GFAP and gelsolin markers in hNSC during differentiation; b) Charts showing the quantitative analysis of the percentage of GFAP- $\delta+$ cells over the total DAPI+ nuclei and of double-stained GFAP-ס/GFAPt+ cells over the total GFAPt+ cells. Statistical significance is indicated. Values are means \pm . Scale bar: $20 \mu \mathrm{m}$. 
A
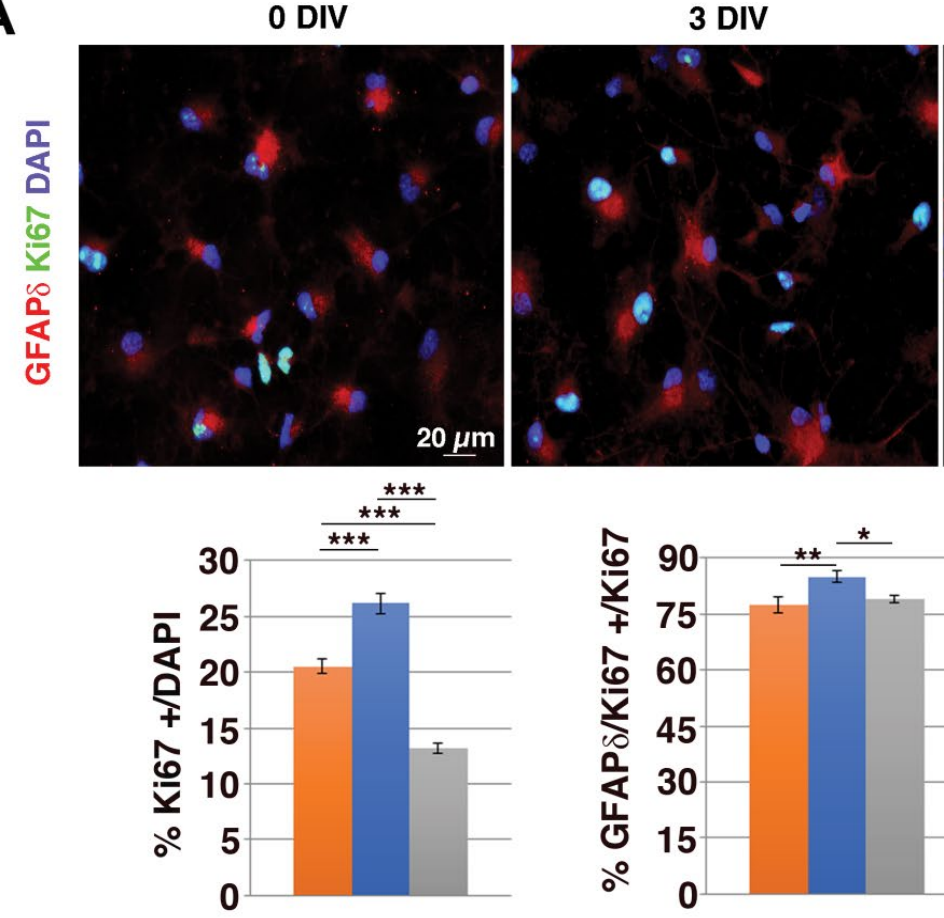

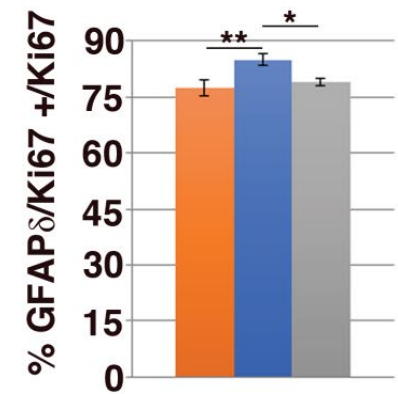

10 DIV
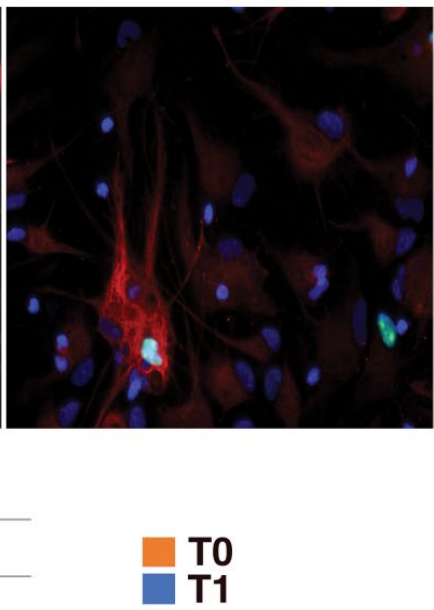

T2

* $p \leq 0.05$

$* * p \leq 0.01$

$\star * \star p \leq 0.001$

B

10 DIV
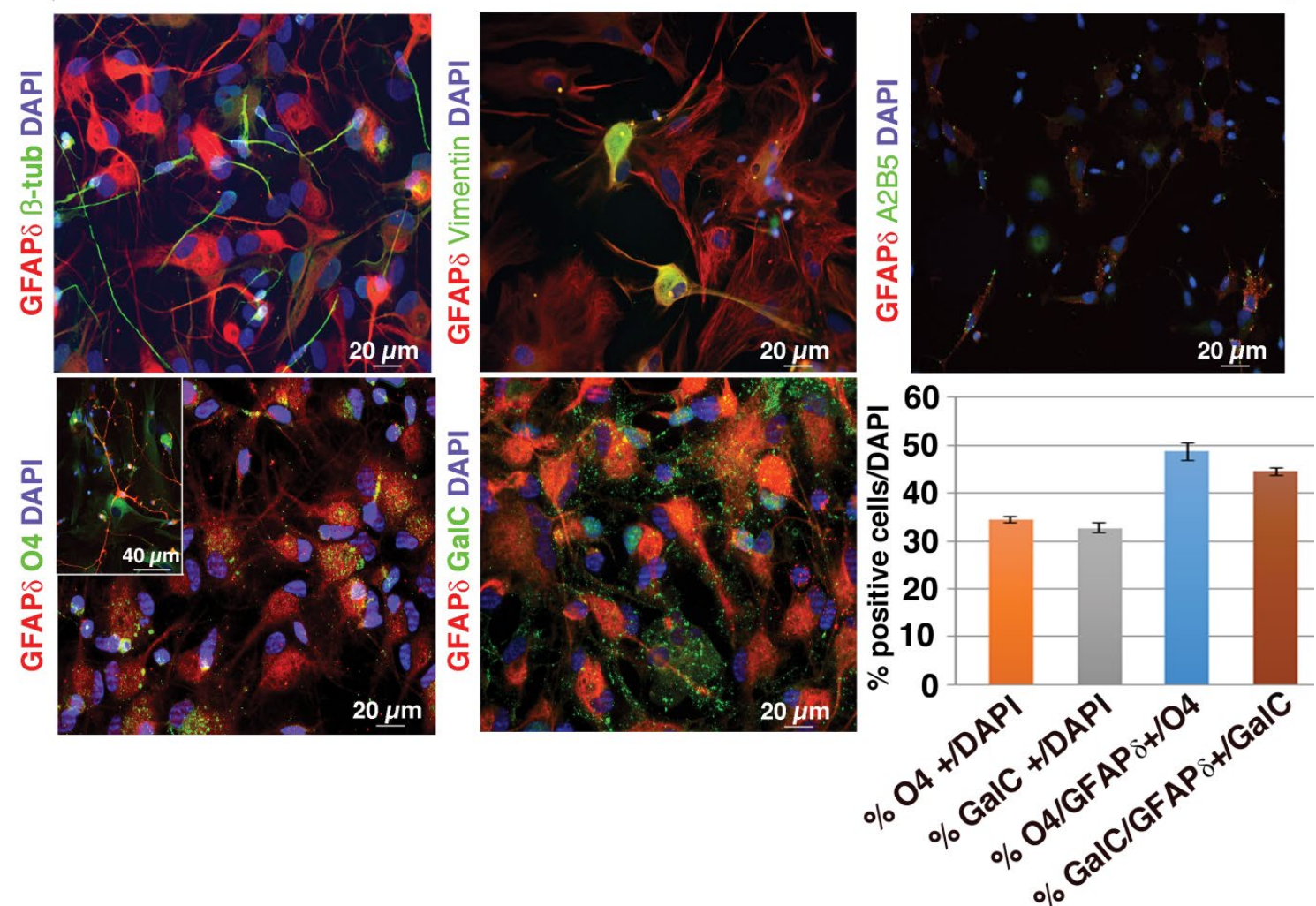

Figure 4: Expression of GFAP- $\delta$ in proliferating glial progenitor cells. a) Immunofluorescence analysis of expression of GFAP- $\delta$ and Ki67 markers in hNSC during differentiation. Charts showing the quantitative analysis of the percentage of Ki67+ cells over the total DAPI+ nuclei and of double-stained GFAP-ס/Ki67+ cells over the total Ki67+ cells. Statistical significance is indicated. Values are means \pm ; b) Immunofluorescence analysis of expression of GFAP- $\delta$, $\beta$-tub, A2B5, Vimentin, O4 and GalC markers in hNSC differentiated at 10 div. Charts showing the quantitative analysis of the percentage of O4+ and GalC+ cells over the total DAPI+ nuclei, of double-stained O4/GFAP- $\delta+$ cells over the total O4+ cells and of double-stained GalC/GFAP- $\delta+$ cells over the total GalC+ cells. Scale bar: $20 \mu \mathrm{m}$. Insert scale bar: $40 \mu \mathrm{m}$. 
Citation: Zalfa C, Grasselli C, Santilli G, Ferrari D, Lamorte G, et al. (2018) GFAP Delta as Divergent Marker of Human Glial Progenitors. Stem Cell Res Ther 8: 437. doi: 10.4172/2157-7633.1000437

Page 7 of 10

as a marker of type II astrocytes and Oligodendroglial Progenitor Cells OPC2A [19]. Interesting, culturing IhNSC under stringent hypoxic conditions ( $1 \%$ oxygen) (Figure $5 \mathrm{a}$ ) led to a re-distribution of the GFAP- $\delta$ protein in proximity of the perinuclear area, that likely corresponds to a stress-induced morphological change compared to physiological hypoxia (5\% oxygen) (Figure $5 \mathrm{~b}$ ). Gelsolin is involved in the control of the organization of the actin cytoskeleton in cells. Indeed, downregulation of gelsolin leads to defective chemotaxis and neurite retraction [20] while its overexpression increases membrane ruffling and chemotaxis [5]. Conversely to GFAP- $\delta$, gelsolin was detected only in astroglial cells (Figure 3a), with a subcellular localization that was periplasmatic at the progenitor stage, but progressively organizing in filaments along with the differentiation process. Interesting, this effect was enhanced under stringent hypoxic conditions (Figure 5a). These results indicated that GFAP- $\delta$ is a marker of stem undifferentiated cells such as of glial progenitors, like gelsolin, but it progressively segregates into specific glial cells over differentiation.

\section{Co-expression of GFAP- $\delta$ and gelsolin in vivo}

On the basis of the results obtained, we hypothesized that GFAP- $\delta$ and gelsolin are both co-expressed by NSCs, thereafter progressively segregating into divergent glial progenitors when NSCs start to differentiate. In a translational view, it is of utmost importance to evaluate the expression of GFAP- $\delta$ and gelsolin in hNSC in vivo, after transplantation into the CNS of acutely injured animals or of animal models of neuroinflammatory diseases. To support our hypothesis that GFAP- $\delta$ and gelsolin are intrinsically expressed by hNSC, we tested their expression in hNSC transplanted into the corpus callosum of focally demyelinated rats at 5 days from lysolecithine-induced lesion [12] (Figure 6a). At 15 days from transplant, human cells were migrating and integrating in the SVZ where they showed to retain coexpression of GFAP- $\delta$ and gelsolin. Interesting, a discrete number of Ki67+ cells were also GFAP- $\delta+$ or gelsolin+, supporting our hypothesis. As expected, in the contralateral non-transplanted emisphere, SVZ endogenous NSC were labelled by gelsolin while no human GFAP- $\delta+$ cell was detectable (Figure 6a, bottom). To exclude that expression of GFAP- $\delta$ and gelsolin was only transiently retained by hNSC after transplantation or induced by acute injury, we analysed hNSC at 3 months from transplantation into the hippocampal fissure of adult rats lesioned by transient global ischemia [14]. While in control unlesioned animals hNSC were poorly surviving and confined to the lesion site (not shown), in lesioned animals hNSC were migrated and integrated in the SVZ, where they retained both GFAP- $\delta$ and gelsolin expression (Figure 6b).

\section{Discussion}

NSCs have been largely studied as of fundamental importance for the therapy of neurodegenerative diseases and currently two main neurogenic regions have been recognized in the adult human brain, the dentate gyrus of hippocampus and the SVZ. For a long time and still to date, the identification of stem cell markers has been a matter of investigation and controversy, but it's commonly accepted that "bona

\section{a}

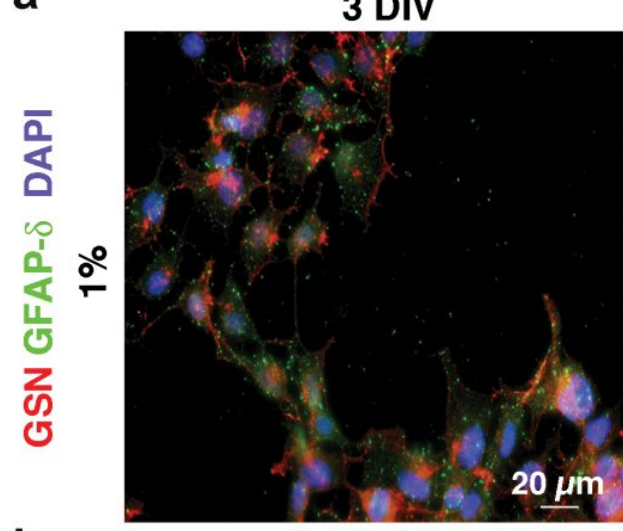

b

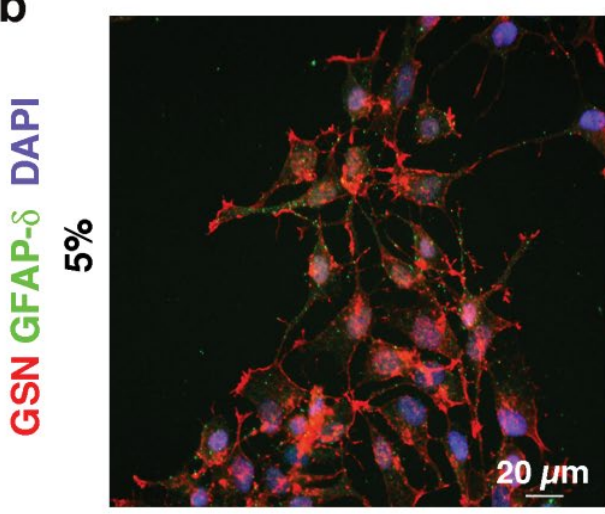

10 DIV
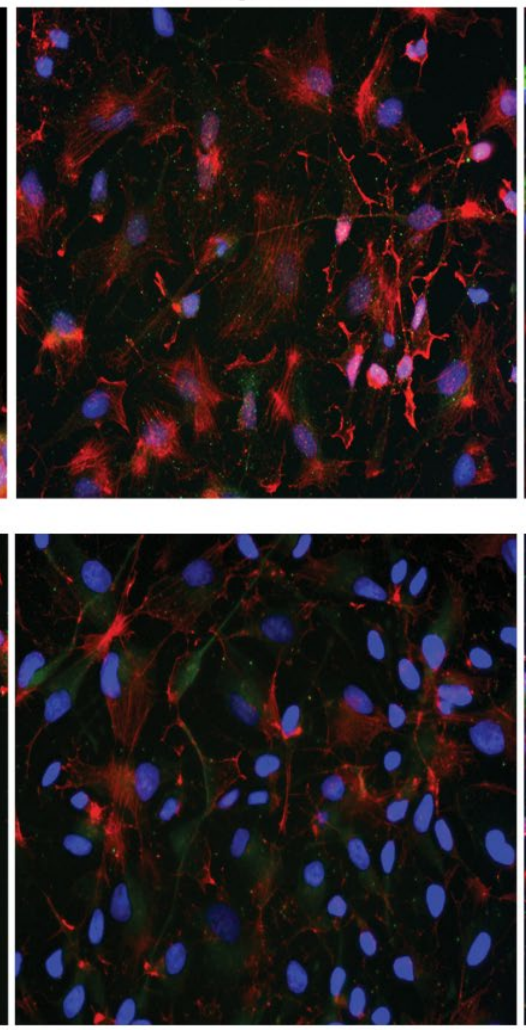

17 DIV
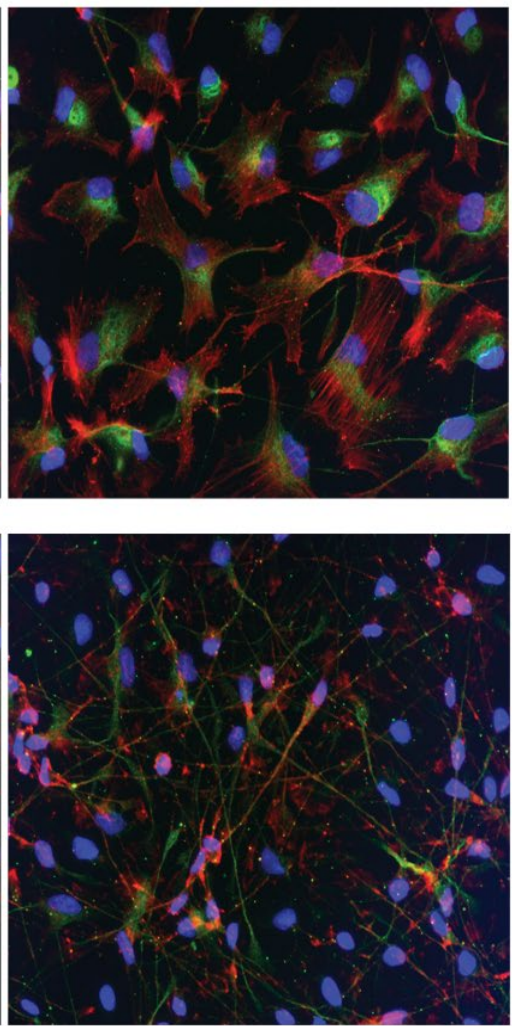

Figure 5: Expression of GFAP- $\delta$ in IhNSC under low oxygen. Immunofluorescence analysis of expression of GFAP- $\delta$ and gelsolin markers in IhNSC during early and late differentiation cultured a) under stringent hypoxic ( $1 \%$ oxygen), and b) normoxic ( $5 \%$ oxygen) conditions. Scale bar: $20 \mu \mathrm{m}$. 
Citation: Zalfa C, Grasselli C, Santilli G, Ferrari D, Lamorte G, et al. (2018) GFAP Delta as Divergent Marker of Human Glial Progenitors. Stem Cell Res Ther 8: 437. doi: 10.4172/2157-7633.1000437

A

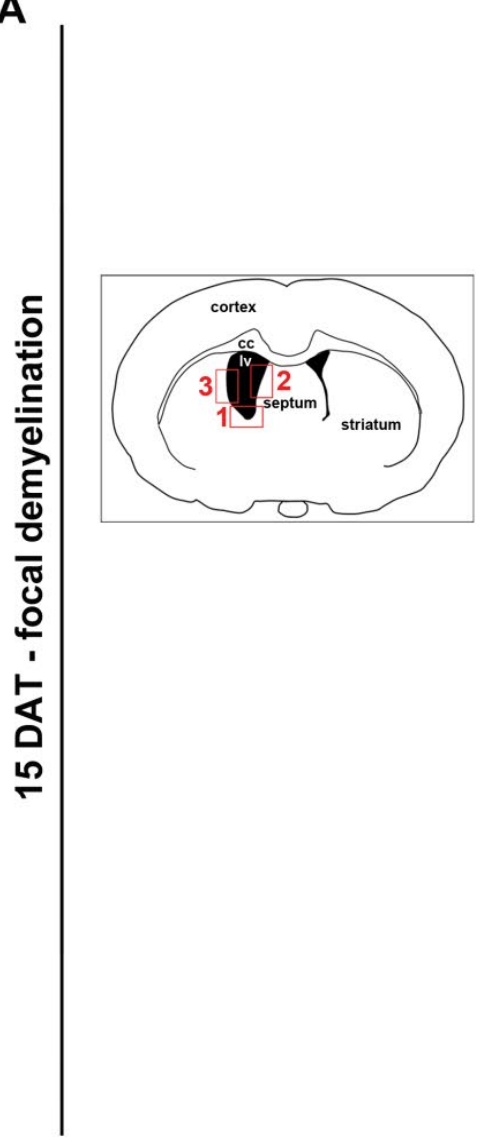

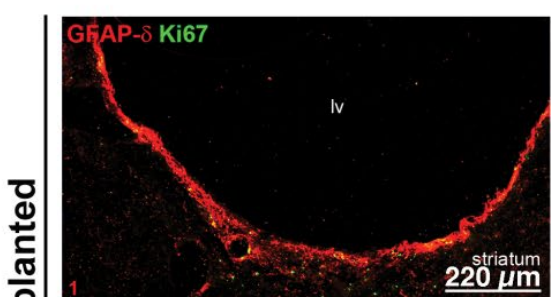
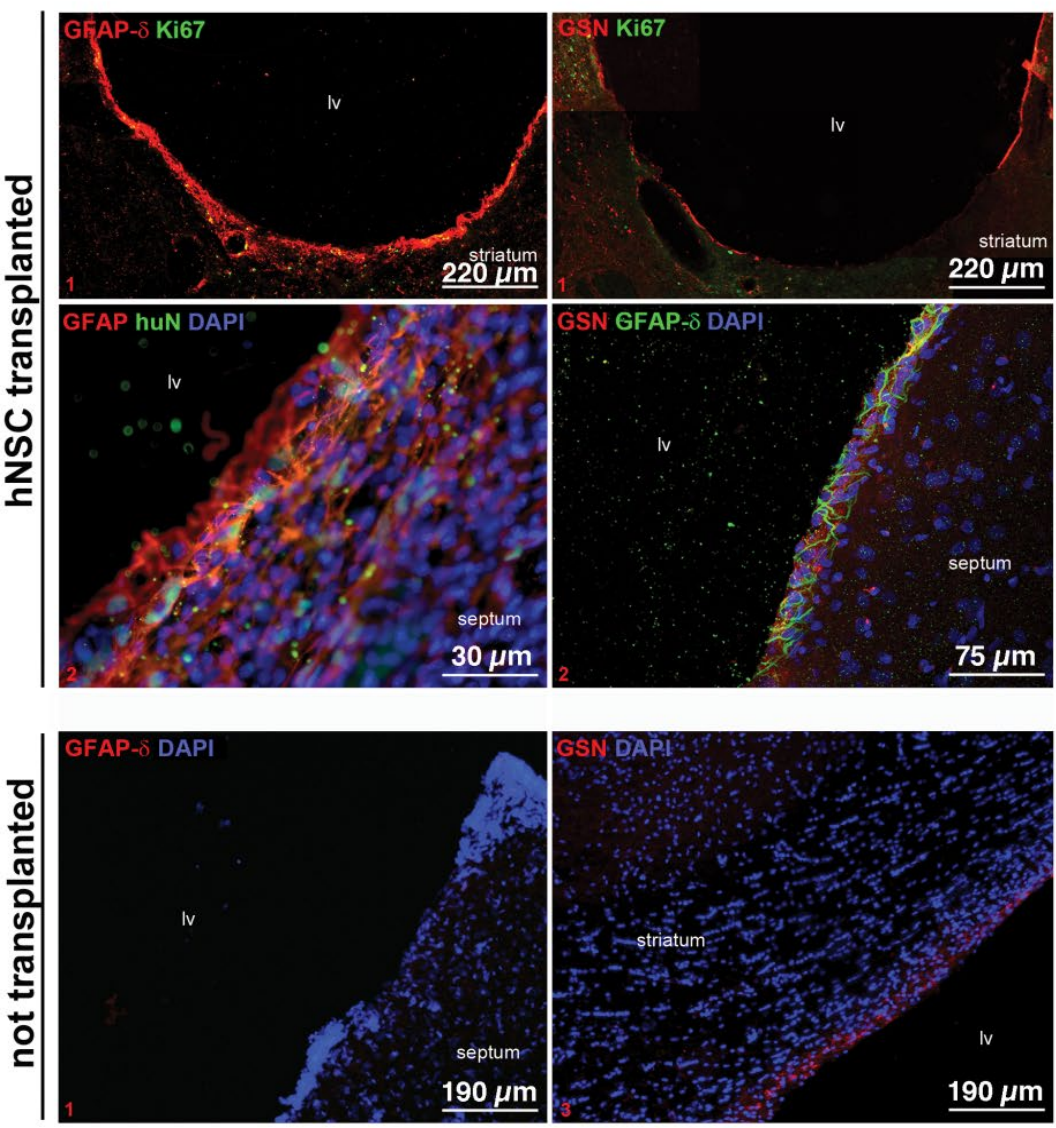

$190 \mu \mathrm{m}$

B
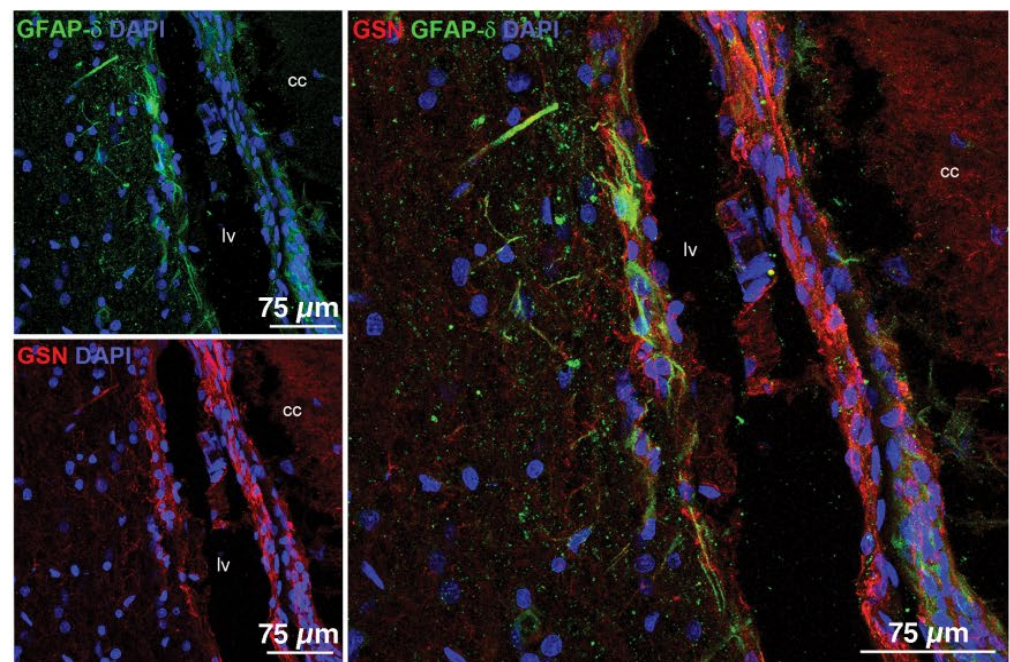

Figure 6: Expression of GFAP- $\delta$ and gelsolin in hNSC in vivo. a) Schematic of a brain coronal section showing hNSCs transplanted into the corpus callosum of focally demyelinated rats at 5 days from lysolecithine-induced lesion and migrating and integrating in the SVZ. Co-expression of GFAP- $\delta$ and gelsolin with Ki67 in hNSCs (upper panel, up), Co-localization of GFAP with huN and GNS with GFAP- $\delta$ is shown in the upper panel, bottom. Human GFAP- $\delta$ and gelsolin expression in the SVZ is also shown in lower panel. Scale bars: $30-220 \mu \mathrm{m}$; b) Schematic of a brain coronal section showing hNSCs transplanted into the hippocampal fissure of an adult rat lesioned by transient global ischemia and migrating and integrating in the SVZ. Expression of GFAP- $\delta$ and gelsolin in hNSCs: both the proteins are expressed by transplanted hNSC but mostly diverge. Scale bar: $75 \mu \mathrm{m}$. 
fide" NSC are GFAP+ [20]. GFAP is a protein component of IFs in astroglial cells and thus participates in regulating their morphology and motility performances. In this view, several studies have investigated cytoskeleton changes involved in the proliferation and migration of NPCs during adult neurogenesis [21]. In particular, the splicing variant of GFAP, GFAP- $\delta$ has been shown specifically expressed in a ribbon below the adult human SVZ and in the SGZ of hippocampus which are commonly recognized as NSC niches and in the subpial cortex where recently active adult neurogenesis has also been demonstrated [2]. Recent findings have elucidated that GFAP- $\delta$ is selectively expressed in a subset of astrocytes corresponding to long-term quiescent cells in the aged human sub-ventricular neurogenic system [2], which are reminiscent of NSCs. Parallel but independent studies have shown that gelsolin, a Ca2+-activated actin-severing protein is widely distributed throughout the adult mammalian brain [3] and is involved in the migration of NPCs with no influence on their proliferation or differentiation capacities, but with important implications on the modulation of exocytotic neurotransmitter release [7]. Thus, considering that both GFAP- $\delta$ and gelsolin are basic components of two different and complementary structures such as respectively IFs and actin filaments, and that both of them seem to intrinsically display complementary effects on NPC self-maintenance or migration to functional brain areas such as cortex and $\mathrm{OB}$, we investigated their expression in human NSC and their differentiated progeny.

We firstly observed the divergence of expression of gelsolin and GFAP- $\delta$ in primary culture of adult cortex, consistently with previous findings showing respectively the expression of gelsolin and of GFAP- $\delta$ in stellate astroglial cells of the sub-pial cortical layer. Then, given the common distribution of the two proteins in the NSCs niches, we looked at their expression in differentiated mixed or astrocyte-enriched cultures of hNSC deriving from the foetal telencephalic-diencephalic region, reminiscent of the adult $S V Z$, or from ventral mesencephalon (IhVMe). Important, in our experiments, we used hNSC produced under GMP regimen and currently exploited as a cell source for transplantation in clinical trial for patients affected by ALS or MS [9] even in this context, displayed divergent localization patterning. Since the proliferation rate of hNSC represents a strong limit to their expansion, we previously obtained hNSC line immortalized with $\mathrm{v}$-myc [8] which is endowed of basal stem properties comparable with parental cells but with enhanced rate of proliferation and self-renewal capacity. Regarding at these cells as a model for actively self-renewing cells, we compared gelsolin and of GFAP- $\delta$ expression patterns in differentiated IhNSC with hNSC progeny: the expression of both the proteins was maintained in the two cellular models. Differently, IVMe differentiated progeny did express gelsolin but not GFAP- $\delta$ showing that the expression of the two proteins is dependent on the intrinsic multipotency and on the brain area of origin of NPCs. Consistently, the expression of the two proteins remained unaltered if the same hNSC line was differentiated into a mixed or astrocyte-enriched progeny. The distinction between different glial progenitor subtypes has been under debate for a long time and still remains unsolved. However, the pattern of GFAP- $\delta$ is consistent with the identification of GFAP- $\delta$ as a candidate marker for either type II astrocyte progenitors (fibroblastlike, vimentin+, GFAP+, A2B5+, O4- and GalC-) or OPC2A (GalC+ and $\mathrm{O} 4+$ ) as described in Miller, et al. [19].

To evaluate to what extent oxygen conditions may regulate gelsolin or GFAP- $\delta$ expression and cause a shift to an astroglioticlike phenotype, we cultured hNSC under normoxic (5\% for the brain) or hypoxic conditions. Under hypoxic conditions, the subcellular distribution of GFAP- $\delta$ was mostly concentrated to the perinuclear area, while no alteration of the protein amount was revealed by Western Blot analysis (not shown), suggesting that GFAP- $\delta$ is not a marker for reactive glia under in vitro stressing conditions.

\section{Conclusion}

GFAP- $\delta$ expression is retained by hNSC that, after transplantation into animal models of focal demyelination or global ischemia, integrate in the SVZ, thus confirming that GFAP- $\delta$ expression is mostly limited to proliferating undifferentiated NPCs in vivo.

Though mostly descriptive, this evidence points to the conclusion that GFAP- $\delta$ and gelsolin both mark proliferating or quiescent hNSC, while over differentiation their differential regulation of IF and actin networks respectively might be preferentially involved in the reorganization of the cytoskeleton in neurogenesis [22] and reactive gliosis [23].

\section{Acknowledgement}

We thank Elly Hol and Jinte Middeldorp for the kind donation of the antibody against the delta isoform of human GFAP- $\delta$ and for the qRT-PCR experiment. The project was funded by ONLUS Foundation Revert.

\section{Disclosure of Interest}

The authors declare that there are no conflicts of interest with respect to the authorship and/or publication of this article.

\section{References}

1. Roelofs RF, Fischer DF, Houtman SH, Sluijs JA, Van Haren W, et al (2005) Adult human subventricular, subgranular, and subpial zones contain astrocytes with a specialized intermediate filament cytoskeleton. Glia 52: 289300. [PubMed]

2. Van den Berge SA, Middeldorp J, Zhang CE, Curtis MA, Leonard BW, et al (2010) Longterm quiescent cells in the aged human subventricular neurogenic system specifically express GFAP-delta. Aging Cell 9: 313-326. [PubMed]

3. Sun HQ, Yamamoto M, Mejillano M, Yin HL (1999) Gelsolin, a multifunctional actin regulatory protein. J Biol Chem 274: 33179-33182. [PubMed]

4. Lu M, Witke W, Kwiatkowski DJ, Kosik KS (1997) Delayed retraction of filopodia in gelsolin null mice. J Cell Biol 138: 1279-1287. [PubMed]

5. Cunningham CC, Stossel TP, Kwiatkowski DJ (1991) Enhanced motility in NIH 3T3 fibroblasts that overexpress gelsolin. Science 251: 1233-1236. [PubMed]

6. Chauhan VP, Ray I, Chauhan A, Wisniewski HM (1999) Binding of gelsolin, a secretory protein, to amyloid beta-protein. Biochem Biophys Res Commun 258 241-246. [PubMed]

7. Kronenberg G, Gertz K, Baldinger T, Kirste I, Eckart S, et al. (2010) Impact of actin filament stabilization on adult hippocampal and olfactory bulb neurogenesis. J Neurosci 30: 3419-3431. [PubMed]

8. De Filippis L, Lamorte G, Snyder EY, Malgaroli A, Vescovi AL (2007) A novel immortal, and multipotent human neural stem cell line generating functional neurons and oligodendrocytes. Stem Cells 25: 2312-2321. [PubMed]

9. Mazzini L, Gelati M, Profico DC, Sgaravizzi G, Projetti Pensi M, et al. (2015) Human neural stem cell transplantation in ALS: initial results from a phase trial. J Transl Med 13: 17. [PubMed]

10. Zalfa C, Verpelli C, D’Avanzo F, Tomanin R, Vicidomini C, et al. (2016) Glia degeneration with oxidative damage drives neuronal demise in MPSI disease. Cell Death Dis 7: 2331. [PubMed]

11. Middeldorp J, Kamphuis W, Sluijs JA, Achoui D, Leenaars CH, et al. (2009) Intermediate filament transcription in astrocytes is repressed by proteasome inhibition. FASEB J 23: 2710-2726. [PubMed]

12. Dijk F, Kamphuis W (2004) An immunocytochemical study on specific amacrine cell subpopulations in the rat retina after ischemia. Brain Res 1026 205-217. [PubMed]

13. Ferrari D, Zalfa C, Nodari LR, Gelati M, Carlessi L, et al. (2012) Differentia pathotropism of non-immortalized and immortalized human neural stem cell lines in a focal demyelination model. Cell Mol Life Sci 69: 1193-1210. [PubMed] 
Citation: Zalfa C, Grasselli C, Santilli G, Ferrari D, Lamorte G, et al. (2018) GFAP Delta as Divergent Marker of Human Glial Progenitors. Stem Cell Res Ther 8: 437. doi: 10.4172/2157-7633.1000437

Page 10 of 10

14. Rota NL, Ferrari D, Giani F, Bossi M, Rodriguez-Menendez V, et al. (2010) Long-term survival of human neural stem cells in the ischemic rat brain upon transient immunosuppression. PLoS One 5: e14035. [PubMed]

15. Nielsen AL, Holm IE, Johansen M, Bonven B, Jørgensen P, et al. (2002) A new splice variant of glial fibrillary acidic protein, GFAP epsilon, interacts with the presenilin proteins. J Biol Chem 277: 29983-29991. [PubMed]

16. Hol EM, Roelofs RF, Moraal E, Sonnemans MA, Sluijs JA, et al. (2003) Neuronal expression of GFAP in patients with Alzheimer pathology and identification of novel GFAP splice forms. Mol Psychiatry 8: 786-796. [PubMed]

17. Vescovi AL, Parati EA, Gritti A, Poulin P, Ferrario M, et al. (1999) Isolation and cloning of multipotential stem cells from the embryonic human CNS and establishment of transplantable human neural stem cell lines by epigenetic stimulation. Exp Neurol 156: 71-83. [PubMed]

18. Doetsch F, Caillé I, Lim DA, García-Verdugo JM, Alvarez-Buylla A (1999) Subventricular zone astrocytes are neural stem cells in the adult mammalian brain. Cell 97: 703-716. [PubMed]
19. Miller RH, Fulton BP, Raff MC (1989) A novel type of glial cell associated with nodes of ranvier in rat optic nerve. Eur J Neurosci 1: 172-178. [PubMed]

20. Witke W, Sharpe AH, Hartwig JH, Azuma T, Stossel TP, et al. (1995) Hemostatic, inflammatory, and fibroblast responses are blunted in mice lacking gelsolin. Cell 81: 41-51. [PubMed]

21. Compagnucci C, Piermarini E, Sferra A, Borghi R, Niceforo A, et al. (2016) Cytoskeletal dynamics during in vitro neurogenesis of induced pluripotent stem cells (iPSCs). Mol Cell Neurosci 77: 113-124. [PubMed]

22. Kamphuis W, Mamber C, Moeton M, Kooijman L, Sluijs JA, et al. (2012) GFAP isoforms in adult mouse brain with a focus on neurogenic astrocytes and reactive astrogliosis in mouse models of Alzheimer disease. PLoS One 7: e42823. [PubMed]

23. Zhang QH, Li JC, Dong N, Tang LM, Zhu XM, et al. (2013) Burn injury induces gelsolin expression and cleavage in the brain of mice. Neuroscience 228: 6072. [PubMed] 